\title{
XMASS 1.5, the next step of the XMASS experiment
}

\author{
Koichi Ichimura*1,2 for the XMASS collaboration \\ ${ }^{1}$ Kamioka Observatory, Institute for Cosmic Ray Research, the University of Tokyo, \\ Higashi-Mozumi, Kamioka, Hida, Gifu 506-1205, Japan, \\ ${ }^{2}$ Kavli Institute for Physics and Mathematics of the Universe, the University of Tokyo, Kashiwa, \\ Chiba 277-8582, Japan \\ E-mail: ichimuradkm.icrr.u-tokyo.ac.jp
}

\begin{abstract}
XMASS, the Kamioka Dark Matter direct detection experiment with an eye to future multi purpose detectors, is planning to improve its Dark Matter sensitivity by increasing the amount of liquid xenon in its inner detector volume from the current $832 \mathrm{~kg}$ to 5 metric tons. Challenges identified with the current detector informed the design of this upgrade. In this presentation, we present the new detector design and discuss how it addresses these challenges. In particular we discuss how new photomultipliers with their non-flat photocathode layout is expected to improve fiducialization.
\end{abstract}

The 34th International Cosmic Ray Conference,

30 July- 6 August, 2015

The Hague, The Netherlands

* Speaker. 


\section{Introduction}

The XMASS project [四] was proposed to observe rare events such as electron recoil from elastic scattering of by solar $p p$ neutrinos, neutrino-less double beta decay, and nuclear recoil from the scattering of dark matter particles with a single phase LXe detector. In the XMASS detector, scintillation light from LXe is observed by photo-multiplier tubes (PMTs) arranged around the LXe volume. LXe is particularly suitable for dark matter searches since it has high atomic number and density. These same properties also allow its use as a shield against external radiation. XMASS-I, the first stage of the XMASS project aims at the search for dark matter. The XMASS-I detector was constructed in 2010 with $835 \mathrm{~kg}$ of LXe in a pentakis-dodecahedral copper vessel, and commissioning runs were conducted from 2010 to 2012. Several physics results were obtained in the commissioning run [[D][目][可][5]. In order to reduce the background found in the commissioning run, the detector was refurbished. After a year of refurbishment work, data taking resumed and is ongoing. The XMASS-I detector is described in detail in Ref. [回].

\section{XMASS1.5}

XMASS 1.5 is the next phase of the XMASS experiment with a total mass of 5 metric tons of liquid xenon. The basic design of the detector is the same as that for the XMASS-I detector. To improve the sensitivity, it is necessary to increase the mass in its fiducial volume, select ultra-low background detector materials and discriminate against background events.

\subsection{Material screening}

The dominant background of the XMASS-I experiment comes from the uranium chain and ${ }^{210} \mathrm{~Pb}$ both in the aluminum seal used in the PMTs. This seal between the body and the quartz window was made with aluminum of standard purity. In the XMASS 1.5 experiment, high purity aluminum is used for this seal in the new PMTs. Furthermore, to achieve our required background level for the new PMT used in the XMASS 1.5 experiment $(0.1 \mathrm{mBq} / \mathrm{PMT}$ for U/Th chain), materials with the lowest possible RI contaminations are selected. Kovar metal which is used for PMT body will be replaced by a Co free metal named 42Alloy. The glass material which is used to insulate the HV pin against this metal body had non-negligible U/Th chain and ${ }^{40} \mathrm{~K}$ RI. For the production of the new PMTs, glass material with lower RI will be used. Such material selection and screening were mainly conducted using 3 HPGe detectors (2 p-type and 1 n-type) and one low background alpha counter at the Kamioka observatory.

\subsection{Distinguish signal from mis-reconstructed surface background}

In the XMASS-I PMTs, photons approaching the PMT from above its entrance window can reach the photocathode. On the other hand, scintillation light emitted from the surface next to this kind of PMT will not be detected on the PMTs right next to where the light was emitted from. Therefore, such surface background events leads to mis-reconstructed events in the fiducial volume, generating background for our rare event searches. To solve this problem, a new type of the PMT which has a dome-shaped photocathode was developed. FigureW shows a picture of the new PMT 
and a schematic representation of the response to surface background event.The dome-shaped PMT can detect the scintillation photons from the neighbor surface background much more effectively than flat PMT.

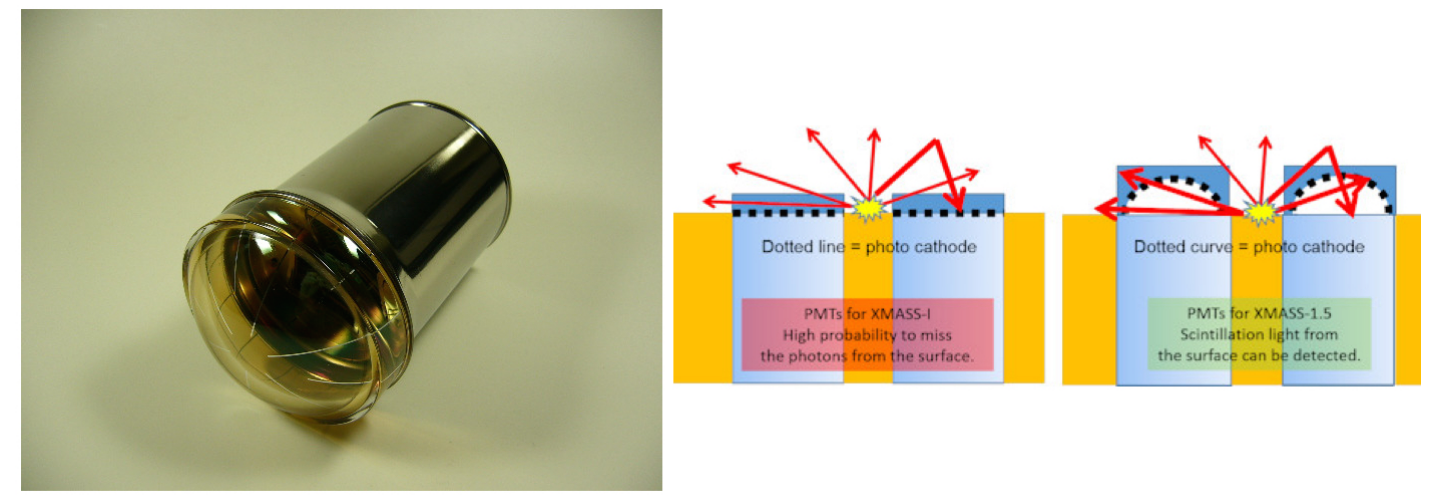

Figure 1: (Left)Picture of the newly-developed 3-inch PMT for XMASS 1.5. (Right)Illustration of the response to surface background of a PMT with a flat photocathode and one with a dome-shaped photocathode.

To demonstrate the performance of the surface background rejection with dome-shaped PMTs, an XMASS-I MC in which the PMTs are replaced with dome-shaped ones is run. Figure $\square$ shows the view from the detector center toward a PMT before and after PMT replacement. Figure $\square$ (right) shows that photons which are emitted in blue the positions hit the dome-shaped photocathode at the red positions. About 50\% of the photons from surface background are detected by the three domeshaped PMTs surrounding the location of a decay. This allows the following parameter named "MaxNPE3 ratio" to be used to distinguish surface background events from events in the fiducial volume.

$$
\text { MaxNPE3 ratio }=\frac{\text { Maximum p.e. in } 3 \text { ad joint PMTs }}{\text { Total observed p.e. }}
$$

Figure[3 shows the comparison between the MaxNPE3 ratio distribution of ${ }^{210} \mathrm{~Pb}$ surface background and that of $2 \mathrm{keV}_{e e}$ alpha events which are uniformly distributed in the liquid xenon volume as signal events. If we cut at 0.1 , more than a factor $10^{5}$ background reduction can be achieved while signal efficiency is still $20 \%$. Further improvement of the analysis will separate signal from

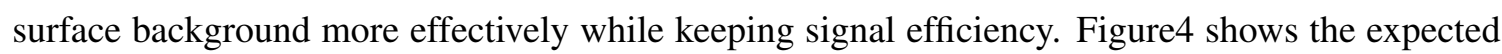
sensitivity for WIMP - nucleon cross sections with the XMASS 1.5 detector and the future XMASS II detector which contains 24 metric tons liquid xenon.Even for the same background level as in the XMASS-I detector, thanks to the dome-shaped PMT and its help in identifying background events from the detector surface, we can search for the heavy WIMPs with cross sections below $10^{-46}$ $\mathrm{cm}^{2}$.

\subsection{Current status for the XMASS 1.5}

The prototype of the dome-shaped PMT has been developed with Hamamatsu. A first batch 

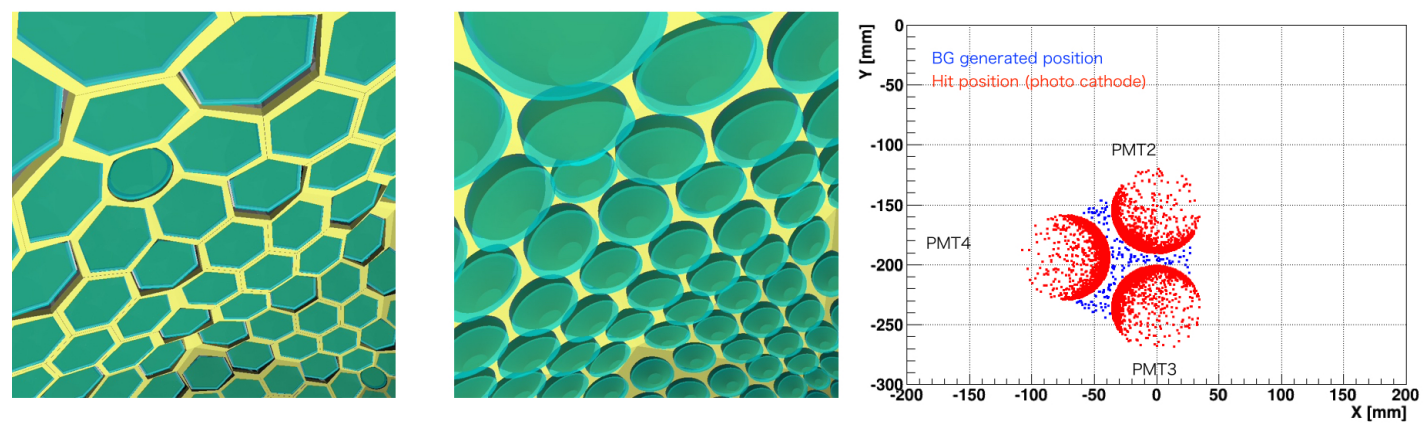

Figure 2: (Left and center) View from the detector center toward PMTs before and after PMT replacement from flat PMT to dome-shaped PMT. (Right)The surface background generation point and photocathode point which detect the scintillation photon from surface background. As can be seen, neighbor 3 PMTs detect many photons from surface background.

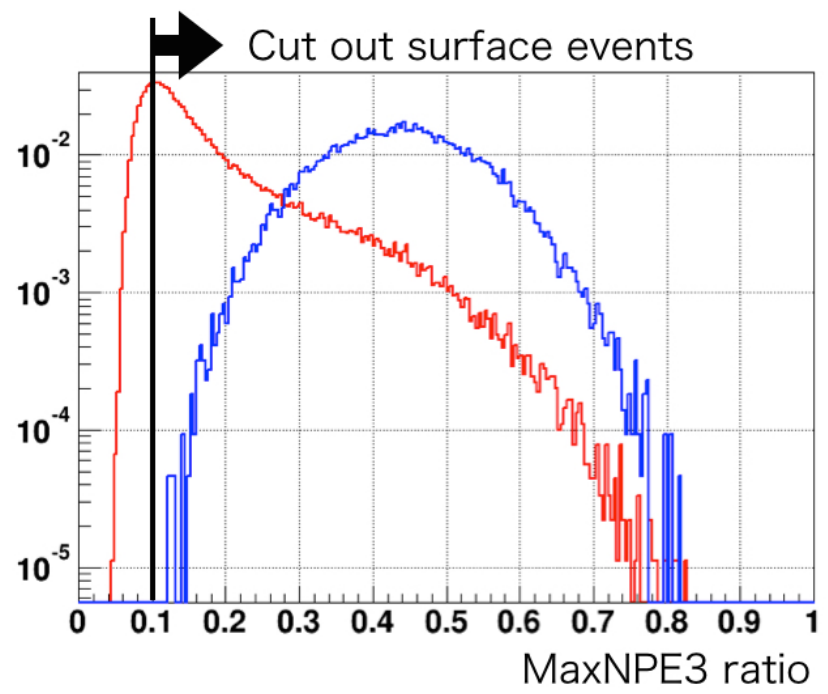

Figure 3: MaxNPE3 ratio distribution of Surface events from ${ }^{210} \mathrm{~Pb}$ (blue) and Uniform signal $\mathrm{MC}\left(2 \mathrm{keV} \mathrm{V}_{e e}\right.$ $\alpha$ events) (red). Both histograms are normalized by area. Surface background MC corresponds to 1.7 year equivalent livetime in case of same background level as XMASS-I detector.

of the new PMTs is currently being tested at the Kamioka Observatory. The measurement of fundamental properties such as PMT gain, transit time spread, long term stability is ongoing. In parallel, material selection and screening for the mass production is continuing. We are studying the XMASS 1.5 performance with these new dome-shaped PMTs using MC simulation, especially the background rejection capability. Currently, PMT arrangement and detector holder geometry are designed in detail. 


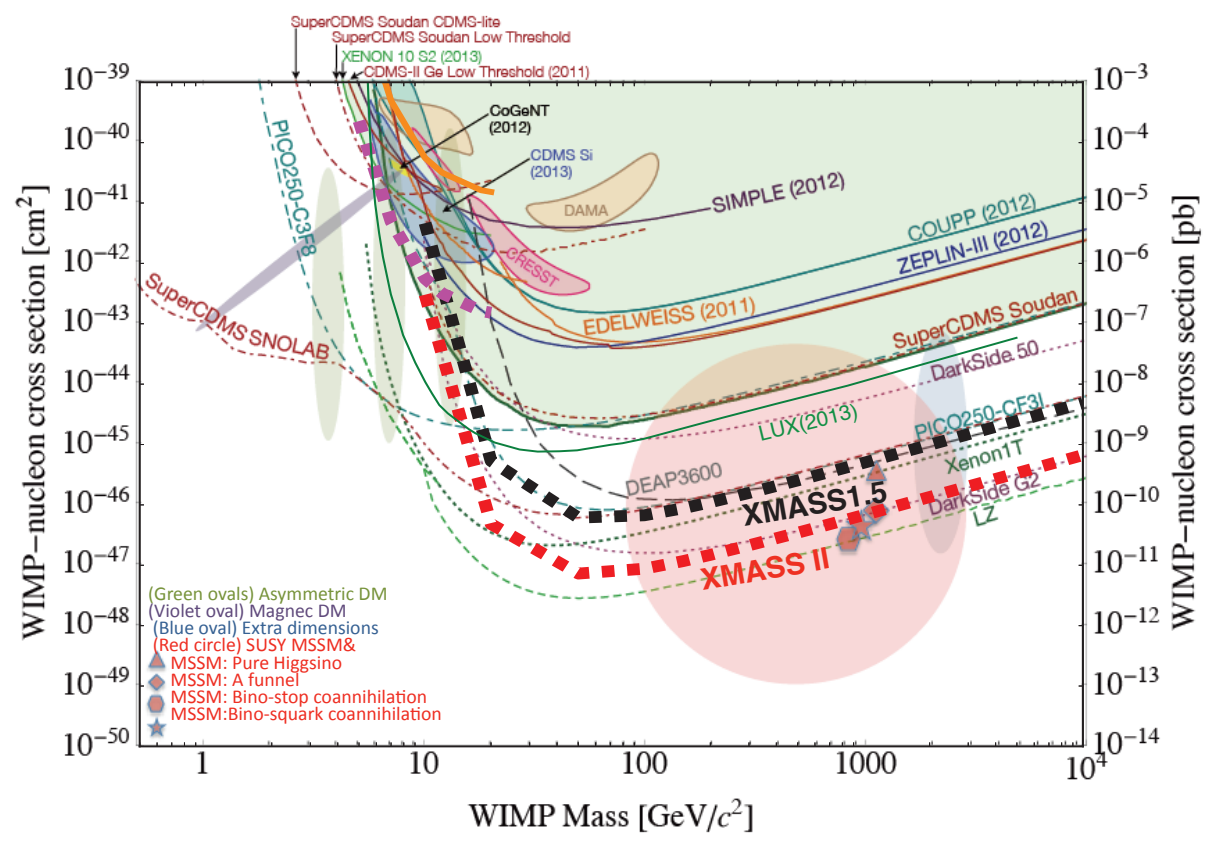

Figure 4: Expected sensitivity with XMASS 1.5 (XMASS-II) assuming fiducial mass of 1 ton (10 tons) and 1 year (5 years) running, respectively

\subsection{Summary}

XMASS 1.5 is the next phase in the XMASS experimental program in Kamioka, Japan. About 900 dome-shaped PMTs and 5 metric tons of liquid xenon will be used for dark matter search. With the new PMTs and carefully selected materials, we expect the sensitivity of this new detector to reach below $10^{-46} \mathrm{~cm}^{2}$ for a $100 \mathrm{GeV}$ WIMP. In addition, XMASS has unique features such as a very low energy threshold and sensitivity to e/ $\gamma$ events, which means that XMASS is sensitive to axion-like particles as well as bosonic super-WIMPs which make electron recoil signal. These features are particularly important to be sensitive to various kinds of the dark matter candidate.

\section{References}

[1] Y. Suzuki, arXiv:hep-ph/0008296.

[2] K. Abe et al., Phys. Lett. B 719, 78-82 (2013)

[3] K. Abe et al., Phys. Lett. B 724, 46-50 (2013)

[4] H. Uchida et al., Prog. Theor. Exp. Phys. 063C01 (2014)

[5] K. Abe et al., Phys. Rev. Lett. 113, 121301 (2014)

[6] K. Abe et al., Nucl. Instr. Meth. A 716, 78-85 (2013) 